\title{
Weather Prediction Improvement Using Advanced Satellite Technology
}

\author{
F. Einaudi , L. Uccellini ${ }^{\dagger}$, J. Purdom $^{* *}$, D. Rogers ${ }^{\dagger \dagger}$, R. Gelaro ${ }^{*}$, J. Dodge $^{\#}$, R. Atlas ${ }^{*}$ and S. Lord ${ }^{\dagger}$ \\ * NASA/Data Assimilation Office, Goddard Space Flight Center, Greenbelt, MD USA \\ †NOAA/NWS/National Centers for Environmental Prediction, Camp Springs, MD USA \\ \#NASA Headquarters, Washington D. C. USA \\ **NOAA/National Environmental Satellite Data and Information Service, Camp Springs, MD USA \\ $\dagger \dagger$ NOAA/Office of Oceanic and Atmospheric Research, Silver Spring, MD USA
}

\section{INTRODUCTION}

We discuss in this paper some of the problems that exist today. in the full utilization of satellite data to improve weather forecasts and we propose specific recommendations to solve them. This discussion can be viewed as an aspect of the general debate on how best to organize the transition from research to operational satellites and how to evaluate the impact of a research instrument on numerical weather predictions. A method for providing this transition is offered by the National Polar-Orbiting Operational Environmental Satellite System (NPOESS) Preparatory Project (NPP). This mission will bridge the time between the present NOAA and Department of Defense (DOD) polar orbiting missions and the initiation of the converged NPOESS series and will evaluate some of the Earth Observing System (EOS) instruments as appropriate for operational missions. Thus, this mission can be viewed as an effort to meet the operational requirements of NOAA and DOD and the research requirements of NASA.

More generally, however, it can be said that the process of going from the conception of new, more advanced instruments to their operational implementation and full utilization by the weather forecast communities is not optimal. Instruments developed for research purposes may have insufficient funding to explore their potential operational capabilities. Furthermore, instrument development programs designed for operational satellites typically have insufficient funding for assimilation algorithms needed to transform the satellite observations into data that can be used by sophisticated global weather forecast models. As a result, years often go by before satellite data are efficiently used for operational forecasts.

NASA and NOAA each have unique expertise in the design of satellite instruments, their use for basic and applied research and their utilization in weather and climate research. At a time of limited resources, the two agencies must combine their efforts to work toward common goals of full utilization of satellite data. This is a challenge that requires the assimilation of myriad new data into increasingly sophisticated numerical forecast models that run on increasingly sophisticated computer systems.

In section II, we briefly outline the impact of satellite data on the quality of the National Centers for Environmental Prediction (NCEP) forecasts. In section III, we describe the present status of the utilization of satellite data in NCEP models and the challenges that lie ahead. In section IV, we propose solutions whose goals are summarized in section $\mathrm{V}$.

\section{STATUS OF SATELLITE DATA IN NCEP MODELS}

Much progress has been made in the utilization of satellite data since the first meteorological satellite was launched in April 1960. During the 1960's and 1970's, enhanced observational coverage preceded the computing capacity and sophisticated assimilation algorithms required to use these data effectively. A seminal advancement related to the direct insertion of satellite radiance data into the models did not occur until 1995 with the inclusion of TOVS cloud-cleared radiances. We might note that the 36 -hr mean sea level pressure forecasts over North America went from having no skill in 1960 (i.e., being no more useful than a forecast based on climatology), to being skillful $72 \%$ of the time in 1995 with the inclusion of TOVS radiances and the use of more sophisticated models on more powerful computers. The horizontal resolution of the models has increased from 381 $\mathrm{km}$ in 1960 to $70 \mathrm{~km}$ today. The vertical resolution has also increased markedly from 1 layer in 1960 to 42 layers today. The computer power, measured in floating point operations per second (flops), has increased from 20 Kflops in 1960 to over 30 Gflops today.

A measure of the impact of satellite data on improving

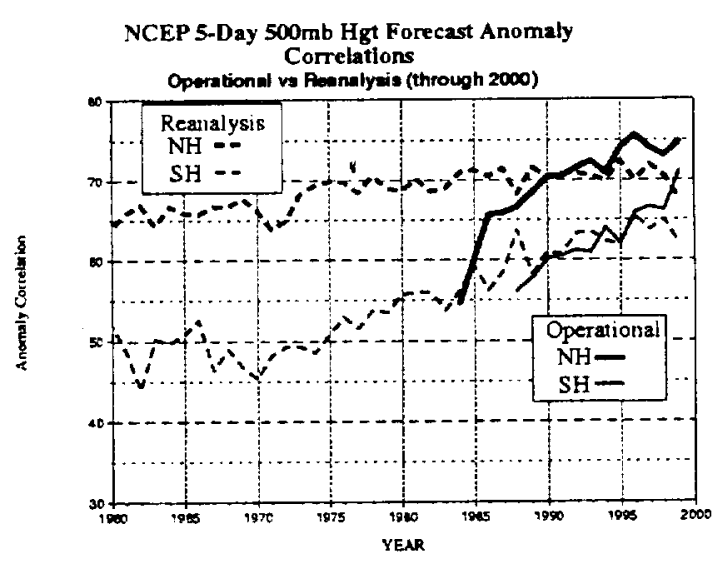

Fig. 1. Anomaly correlation coefficient (ACC) for $500 \mathrm{mb}$ height for the NCEP 5-day forecast as a function of time. Dashed (solid) lines refer to fixed (evolving) model and assimilation system. Bold (thin) lines refer to Northem (Southern) Hemisphere. 
operational numerical weather forecasts is given in Fig. 1, which shows the anomaly correlation coefficient (ACC) for $500 \mathrm{mb}$ height calculated for the NCEP 5-day forecast as a function of time. The correlation is between the observed and predicted deviations from the climatological $500 \mathrm{mb}$ height field. The dashed lines show the ACC calculated while reprocessing all the data with the model and data assimilation system kept fixed at the 1995 configuration. Results are shown for the Northern (bold) and Southern (thin) Hemispheres. Neglecting interannual variability, a steady improvement in the ACC is evident, with a larger rate of improvement for the Southern Hemisphere. The improvement must be due to new data, with the majority of new data coming from polar satellites. The larger rate of improvement for the Southern Hemisphere provides further evidence of the importance of satellite data, given the relative paucity of insitu data there.

The solid lines in Fig. 1 show the ACC when the data are processed with an evolving operational system. The noticeably large improvements in 1996 and 1999 are due, in large part, to the introduction of direct assimilation of the radiances and inclusion of Advanced Microwave Sounding Unit (AMSU) data, respectively.

In addition to operational satellites, research instruments have also demonstrated positive impact in weather forecast models. An example of a research instrument that has shown tremendous potential operational impact is the scatterometer, which measures wind speed and direction over the global oceans. First flown on ERS-1 in 1991, this instrument presently flies on Quikscat, launched in 1999. The impact of Quikscat data was tested for several numerical forecasts of hurricanes over the Atlantic Ocean. For Hurricane Cindy in August 1999, the inclusion of Quickscat data reduced intensity errors by nearly $50 \%$, and displacement errors by up to $80 \%$, over a 72 -hr forecast (Fig. 2). Improvements of this order are critical to reducing the uncertainty in hurricane track and intensity forecasts needed to allow emergency managers to make informed decisions.

\section{THE NATURE OF THE PROBLEM}

The transition from research and development (R\&D) to operations is a complex topic that has been discussed in a number of reports. Reference [1] discusses this issue vividly and refers to the term "Crossing the Valley of Death" as being used in industry to describe a fundamental challenge for R\&D programs. Moreover, [1] states that "For technology investments, the transitions from development to implementation are frequently difficult, and, if done improperly, these transitions often result in "skeletons in Death Valley." Successful transitions from R\&D to operational implementation always require: (1) an understanding of the importance (and risks) of the transition, (2) development and maintenance of appropriate transition plans, (3) adequate resource provision, and (4) continuous
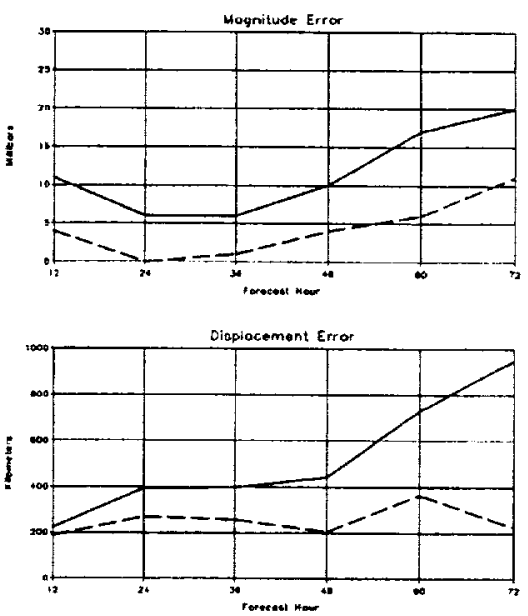

Fig. 2. Forecast errors in terms of (a) intensity and (b) position for Hurricane Cindy with (dashed) and without (solid) assimilation of Quickscat data. Units are $\mathrm{mb}$ in (a) and $\mathrm{km}$ in (b).

feedback (in both directions) between $R \& D$ and operational activities. In the case of atmospheric and climate sciences, inadequacies in transition planning and resource commitment can seriously inhibit the implementation of good research leading to useful societal benefits."

The situation is particularly serious for satellite data utilization. Although satellite data account for nearly $83 \%$ of the data assimilated in the NCEP models today, these data only correspond to roughly $14 \%$ of the available satellite data from operational lower-Earth orbiting systems. Most data are thinned upon receipt to minimize cross-correlation errors during ingestion. Furthermore, radiance data collected over land cannot be used directly because of the difficulties in modeling the varying emissivity and associated surface characteristics.

The problem is compounded by the five-order magnitude increase in satellite data expected in the next ten years. Fig. 3 shows the number of individual daily upper air observations used by the NCEP operational models as a function of time, and an estimate of the data volume by 2010 . Correspondingly, Fig. 4 shows a partial list of the new operational satellite instruments planned for launch over the next ten years that account for the five-order magnitude increase in available data. We are thus faced with a situation where current satellite data are under utilized operationally, and a new, more voluminous set of data will be available within ten years.

The present situation is the consequence of many factors:

- Insufficient funding for the operational assessment of instruments developed for research purposes.

- Insufficient funding for assimilation algorithms for instruments developed for operational satellites.

- Tendency of the operational community to be restrictive in the use of data from research satellites that may not be sustained as operational systems.

- Tendency of the operational community to be slow in the 


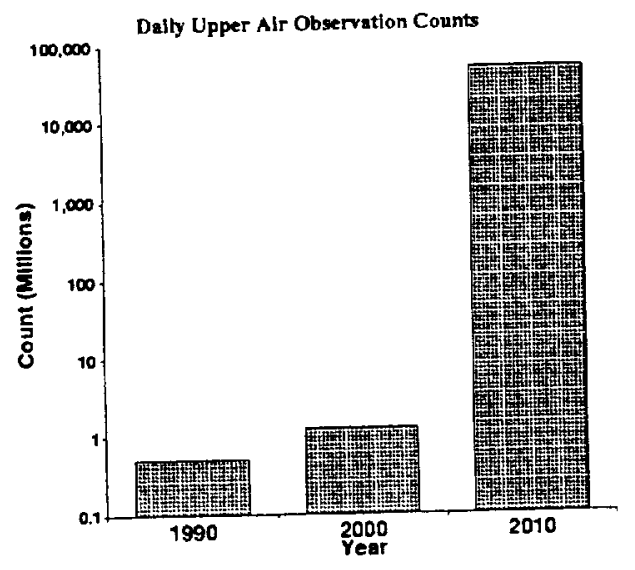

Fig. 3. Current and expected number (in millions) of daily upper air observations used by NCEP models as a function of time.

assessment and incorporation of new data.

- Underutilization of the full breadth of the weather research community in support of operational problems.

As a result, there often is a gap of many years before data are fully used operationally, as in the case of the radiance data from the GOES sounder. The situation has improved recently with the example of AMSU data being used by NCEP within 10 months of launch. However, we can and must do better to capitalize fully on the investment the U.S. makes on its satellite observing network.

To address these problems, NASA and NOAA scientists and managers should be involved in an end-to-end process, from defining an instrument, to characterizing that instrument's in flight performance, to developing algorithms and forward models for data assimilation, to integrating the data into NOAA operational products, to assessing its impact on the forecast.

To cover the entire process, the two agencies must work together and share costs for the full spectrum of these activities. Presently, this comprehensive approach is not taking place and areas of interaction involve only selected portions of the end-to-end process defined above. This need for comprehensive collaboration is the fundamental issue that the two agencies must address. Moreover, this collaboration is consistent with the strategic plans and vision statements of both agencies and should facilitate major advances in the analysis and prediction of weather, climate and hydrology. Accordingly, each agency would have its own role:

- NASA would apply scientific and technological capabilities that support NOAA's goals and operational forecast responsibilities.

- NOAA would apply operational model infrastructure and applied research capabilities that support NASA's research and technology-advancement objectives.

\section{SPECIFIC RECOMMENDATIONS}

To achieve the goal of fully utilizing satellite data for both

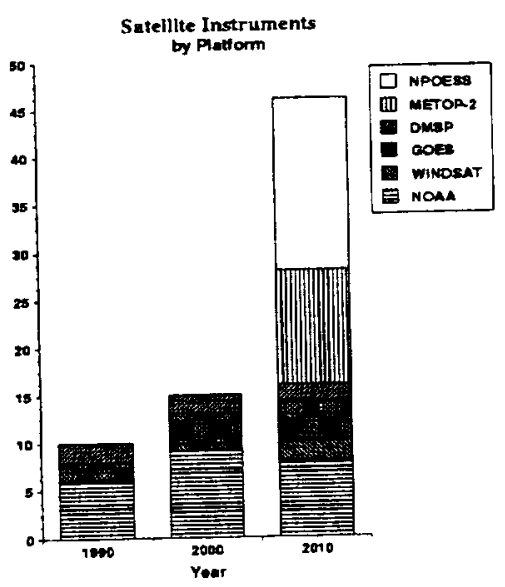

Fig. 4. Partial list of current and expected operational satellite instruments as a function of time.

research and operations, we propose a collaborative effort where NASA and NOAA direct necessary resources to accomplish the following.

\section{Support the goals of the U.S. Weather Research Program (USWRP) to:}

a. increase understanding and prediction of Hurricanes at Landfall (HAL);

b. improve Quantitative Precipitation Forecasts (QPF);

c. extend accurate weather forecasts to 7 days and beyond.

\section{Establish a NASA-NOAA Joint Center for Satellite Data} Assimilation (JCSDA) to:

a. accelerate the use of data from advanced satellite sensors being launched into space by NOAA, NASA, DOD and international agencies;

b. develop and maintain a common model and data assimilation infrastructure for optimal use of satellite observations for research and operational applications;

c. develop and maintain a fast forward radiative transfer model for use of satellite microwave and infrared sounding data in data assimilation and product generation;

d. assess the use of satellite data within the common model and data assimilation infrastructure.

3. Develop an end-to-end process for the operational utilization of polar and geostationary observations.

This collaborative approach represents a commitment of NASA and NOAA to focus on research and operational issues related to improving the forecast of high impact weather events and extending accurate weather forecasts to seven days and beyond. Furthermore, these actions will accelerate the use of existing and new satellite data by the research and operational communities.

The USWRP addresses key issues related to the highest priority goals of the weather prediction component of the National Weather Service (NWS) strategic plan. NASA is interested in many components of the USWRP for several 
reasons: (1) NASA's Earth Sciences Enterprise program has scientific and technological components relevant to forecasting extreme weather events and extending accurate weather forecasts to seven days and beyond, (2) USWRP provides an opportunity to integrate research data sets from instrumented aircraft within multi-agency field programs, (3) USWRP provides an excellent opportunity to assess the utility of various satellite observing systems in forecast models and (4) USWRP makes significant strides in establishing the credibility of global/climate models for predicting short range weather systems and thus assuring a physical consistency of these models. These consistencies will ease the transfer of physical parameterization schemes between global and mesoscale models.

The NASA-NOAA JCSDA would be a virtual center which will engage the Goddard Data Assimilation Office (DAO), the NESDIS Office of Research and Applications (ORA), the Office of Oceanic and Atmospheric Research (OAR) Forecast Systems Laboratory (FSL) and Environmental Technology Laboratory (ETL), and the NCEP Environmental Modeling Center (EMC) in a determined effort to ingest and use the large volume of data associated with planned satellite missions over the next 10 years. The advanced instruments from planned NASA and NOAA missions will provide data on atmospheric, oceanic and land surface conditions with accuracies and spatial resolutions never before achieved. Without the establishment of a NASA-NOAA JCSDA that focuses on satellite data assimilation, the nation's ability to utilize fully current and planned satellite observing systems will fall further behind over the next five to ten years as new generations of sensors are flown by NOAA, NASA, DOD and international agencies. Advances that might be realized from EOS, New Millennium Program (NMP), NPOESS NPP and NPOESS itself will not be realized by the NWS until well into the lifetimes of those programs, if ever. The establishment of a NASA-NOAA JCSDA must be recognized as a NASA and NOAA imperative to ensure that the nation realizes the maximum benefit from its investment in space as part of an advanced global observing system.

The JCSDA should be started from existing scientific interactions between NASA/DAO and NOAA/NCEP. These interactions have already produced a 13-point plan focused on testing existing and new satellite observations within the operational modeling framework, including data from AMSU, Quikscat, Tropical Rainfall Measurement Mission (TRMM), and GOES.

The JCSDA will have minimal bureaucratic infrastructure and will make a determined effort to engage the community at large. It is hoped that the National Science Foundation will support students and post-doctoral fellows who would perform their work at the Center.

\section{GOALS AND EXPECTED DELIVERABLES}

JCSDA activities will be divided into infrastructure activities and proposal-driven scientific projects. Initially, infrastructure activity will focus on the development and maintenance of a scientific backbone for the JCSDA composed of a community-based fast radiative transfer model, a community-based emissivity model and an infrastructure for performing assimilation experiments with real and simulated observations from new and future instruments. Proposal-driven scientific projects will be the primary mechanism used to accelerate the transition of research and technological advances in satellite data assimilation by incorporating new codes into NASA and NOAA operational data assimilation systems and by performing preliminary testing with these systems. Initial JCSDA projects will serve to solidify existing collaborations on AIRS, QuikSCAT and TRMM, and to develop new collaborations in areas deemed potentially important for improving climate and weather prediction but where current activity is relatively weak, such as in the utilization of GIFTS, VIIRS and GPS radio-occultation data.

A primary goal of the JCSDA in the time frame 2001-2003 will be to lay the groundwork for establishing a common data assimilation infrastructure for assessing new satellite data and optimizing the utilization of these data in operational models. The first step is to establish, and make accessible to the community at large, parallel versions of the NCEP/EMC and NASA/DAO global data assimilation systems on JCSDA computer systems. This will include establishment of realtime communications to JCSDA computers and real-time data bases and observation handling algorithms for continued assessment of new instruments.

JCSDA deliverables by 2005 will include the development of a community-based forecast and data assimilation system for both global and regional scale applications. The system will be linked to the research community through the USWRP and will serve as the primary mechanism for infusing research and operational satellite data into NCEP operations.

The JCSDA thus provides a bridge for NASA and NOAA to act on new satellite observing systems, and a way forward for achieving both agencies' vision of improving climate and weather prediction using advanced satellite technology.

\section{ACKNOWLEDGMENT}

The authors are grateful to R. Kistler and J. Terry for their efforts in preparing the figures for this paper.

\section{REFERENCES}

[1] National Research Council, Board on Atmospheric Sciences and Climate, 2000: From Research to Operations in Weather Satellites and Numerical Weather Prediction: Crossing the Valley of Death. National Academy Press, 80 pp. 